\title{
Diversifying the platinum-based chemotherapy toolkit for immunogenic cancer cell death
}

\section{Abhishek D. Garg and Patrizia Agostinis}

Platinum-based chemotherapeutics, such as cisplatin, oxaliplatin and carboplatin, are amongst the most widely applied anticancer agents, with wellestablished efficacy against several cancer-types [1, 2]. In fact, in few clinical settings (e.g., loco-regionally advanced cancer and metastatic germ cell cancers), platinum-based chemotherapy has exhibited promising curative effects, when combined with other conventional anticancer modalities [1]. The binding of reactive platinum molecules derived from these chemotherapies (after they enter the cells) to DNA, thereby creating potent platinumDNA adducts, is widely considered to be the primary modus operandi behind their anticancer activity [3]. Such adducts interfere with normal DNA turn-over and repair machinery, thereby inducing regulated cell death (RCD) mainly in the form of apoptosis [3].

For a long-time it was considered that the mechanistic underpinning of the anticancer efficacy of such platinum-based chemotherapies originated from their ability to induced DNA damage causing cancer cell death [3]. However, over the last decade, it has been repeatedly documented that these platinum-based chemotherapies can also enhance the immunogenicity of dying cancer cells by inducing immunogenic cell death (ICD), in a context-dependent fashion [4]. ICD is a form of (apoptotic or necroptotic) RCD that exhibits immunogenic characteristics due to the exposure and delivery of various immunomodulatory signals e.g., damage-associated molecular patterns (DAMPs), which stimulate the innate immune cells to prime adaptive immune responses against tumor antigens $[4,5]$. Cancer cells undergoing ICD can elicit potent anticancer immunity, predominantly in cancer cells enriched in carcinogen-induced antigens [5]. Interestingly, qualitative as well as quantitative differences have been observed between different platinum-based chemotherapies in terms of ICD-induction capacities [4]. For instance, while oxaliplatin has the most consistent ICD-inducing capacity yet cisplatin/carboplatin tend to induce largely non-ICD characteristics [4]. Although in some exceptional instances, cisplatin can also exhibit ICD-like activity (the mechanistic basis of which, is asyet unclear) [6]. Nevertheless, a clear chemical structurefunction relationship between these agents and ICDinduction has not been established thereby mandating systematic testing of all platinum-based chemotherapies in order to expand the ICD toolkit. Interestingly, several new platinum-based chemotherapeutic agents are currently entering clinical oncological trials. One such promising agent is R,R-1,2 cyclohexanediamine-pyrophosphatoplatinum(II) (PT-112) [7]. PT-112, owing to a more robust chemical structure, has better pharmacokinetic/ pharmacodynamic properties compared to currently used platinum-based chemotherapeutics, which accounts for its improved anticancer effects [7]. In line with this, preliminary measurements of clinical benefit in cancer patients resistant to various conventional and/or experimental modalities, showed some early (therapeutic) benefits upon PT-112 treatment within ongoing Phase I clinical trials (NCT02266745, NCT03409458) - although these observations need to be further verified in advanced clinical trials [7]. Nevertheless, PT-112 monotherapy was also reported to exhibit anticancer efficacy in two patients with resistance toward immunotherapy [7]. Although these observations underscore the promising anticancer efficacy of PT-112 yet they also hint toward its possible pro-immunogenic activity. However, little is known about the cell death-associated immunogenic characteristics of PT-112 in the setting of anticancer therapy.

Interestingly, a recent study by Yamazaki et al. tried to address this gap-in-knowledge [7]. Yamazaki et al. documented that PT-112 exhibits promising in vitro cell death-inducing activity against several human and murine cancer cell lines [7]. In line with a ICD-like profile, the cell death induced by PT-112 associated with the main ICD-associated DAMPs like calreticulin surface exposure, ATP secretion and passive HMGB1 release [7]. Moreover, Yamazaki et al. were able to successfully demonstrate that cancer cells dying in response to PT-112 in vitro, induced anticancer vaccination effect in vivo; i.e., when PT-112 treated dying/dead cancer cells were injected as "vaccines" in syngeneic, immunocompetent mice, these mice developed immunological immunity against a consequent challenge with live cancer cells of identicaltype [7]. These results establish PT-112 as a new platinumbased chemotherapeutic with ICD-inducing capacity.

Over the years, immunotherapy involving immunecheckpoint blockers (ICBs) that inhibit immunecheckpoint signalling downstream of PD1/PD-L1 or CTLA4, has become standard-of-care for several cancertypes [8]. Hence currently, it is a 'hot area' of investigation to characterize chemotherapies that can (or cannot) synergize with ICBs. Importantly, at least one clinical trial is currently exploring combination of PT-112 with the PD-L1 targeting ICB, Avelumab (NCT03409458), 
thereby making it imperative to preclinically test the immunological effects of the combination of PT-112 with ICB. To address this, Yamazaki et al. administered tumour-bearing mice with PT-112 in combination with different ICBs and found that PT-112 synergizes with ICBs targeting PD1 or PD-L1 in reducing tumour burden (in immune-competent settings) [7]. These anticancer effects in combinatorial settings were accompanied by intra-tumoural recruitment of various effector immune cells paralleling a reduction in immunosuppressive cells [7]. Interestingly, the combination of these ICBs with PT112 also exhibited "abscopal effect" i.e., treatment of a particular tumour reduced the growth of another distant (untreated) tumour [7].

Altogether these results demonstrate that, PT-112 not only exerts pro-immunogenic effects via ICD but can also act as a promising combinatorial agent for ICBbased immunotherapy. Of note, the in vivo immunological assays in this study were mainly performed with relatively immunogenic cancer models like TS/A murine breast cancer cells or CT26/MC38 murine colon cancer cells, that have been widely used to study and annotate ICD [7]. In future it would be interesting to explore how PT112 performs against highly immunoevasive or negligibly immunogenic cancer models where standard ICD frequently fails. Additionally, further investigations on the molecular underpinnings of the danger signaling pathways elicited by PT-112 would be instrumental in understanding why assorted platinum-based chemotherapies induce ICD, while others like cisplatin, are much less effective. Nevertheless, the observations of Yamazaki et al. are intriguing and create a definitive incentive to further explore the exact cellular and molecular mechanisms underlying their results. Such insights can help in conceptualization of more improved platinum-based chemotherapies with augmented pro-immunogenic effects against tumours.

\section{CONFLICTS OF INTEREST}

Authors have no conflicts of interest to declare.

Abhishek D. Garg: Cell Stress \& Immunity (CSI) Laboratory, Department for Cellular \& Molecular Medicine (CMM), KU Leuven, Belgium

Correspondence to: Abhishek D. Garg,

email abhishek.garg@kuleuven.be

Patrizia Agostinis: Cell Death Research \& Therapy (CDRT) Laboratory, Department for Cellular and Molecular Medicine (CMM), KU Leuven, Leuven, Belgium; Center for Cancer Biology (CCB), VIB, Leuven, Belgium

Correspondence to: Patrizia Agostinis, email patrizia.agostinis@kuleuven.be
Abhishek D. Garg and Patrizia Agostinis are equally contributed to this work.

Keywords: PT-1 12; immunogenic cell death (ICD); immunecheckpoint blockers (ICBs); tumour microenvironment; apoptosis

Received: August 03, 2020

Published: September 08, 2020

\section{REFERENCES}

1. Hato SV, et al. Clin Cancer Res. 2014; 20:2831-2837. https://doi.org/10.1158/1078-0432.CCR-13-3141. [PubMed]

2. Monneret C. Ann Pharm Fr. 2011; 69:286-295. https://doi. org/10.1016/j.pharma.2011.10.001. [PubMed]

3. Wang D, et al. Nat Rev Drug Discov. 2005; 4:307-320. https://doi.org/10.1038/nrd1691. [PubMed]

4. Galluzzi L, et al. J Immunother Cancer. 2020; 8:e00337. https://doi.org/10.1136/jitc-2019-000337. [PubMed]

5. Garg AD, et al. Immunol Rev. 2017; 280:126-148. https:// doi.org/10.1111/imr.12574. [PubMed]

6. Di Blasio S, et al. Oncoimmunology. 2016; 5:e1192739. https://doi.org/10.1080/2162402X.2016.1192739. [PubMed]

7. Yamazaki T, et al. Oncoimmunology. 2020; 9:1721810. https://doi.org/10.1080/2162402X.2020.1721810. [PubMed]

8. Chae YK, et al. J Immunother Cancer. 2018; 6:39. https:// doi.org/10.1186/s40425-018-0349-3. [PubMed]

Copyright: Garg and Agostinis. This is an open-access article distributed under the terms of the Creative Commons Attribution License 3.0 (CC BY 3.0), which permits unrestricted use, distribution, and reproduction in any medium, provided the original author and source are credited. 